\title{
IX. Ueber ein neues Condensations- oder
} Absorptionshygrometer; von A. Matern.

In jüngster Zeit ist von E delmann ein Absorptionshygrometer construirt worden, zu welchem A. van Hasselt ${ }^{1}$ ) einige Verbesserungen vorgeschlagen hat. Wenn auch nach Angabe der genannten Physiker mit diesen Apparaten günstige Resultate sich ergeben haben, so scheinen mir uncontrolirbare Fehler doch nicht ganz ausgeschlossen zu sein.

Fürs erste gilt dieses Bedenken dem Vorhandensein einer zu grossen Zahl von Stellen, an denen Luftzutritt möglich ist und verhindert werden soll.

Ferner sind Temperaturänderungen der im Apparate eingeschlossenen Luft wegen der durch die. Absorption entstehenden Wärme unvermeidlich, können an einem in unbewegter Luft befindlichen Thermometer nur langsam und unsicher ermittelt werden und bewirken an dem gegen die äussere Luft sich öffnenden Manometer beträchtliche Druckänderungen; ein Temperaturunterschied von $\frac{1}{10}$ Grad verursacht eine Druckdifferenz von $0,27 \mathrm{~mm}$, also eine im Verhältniss zu dem gesuchten Dunstdruck oft recht erhebliche Grösse.

Zur Absorption benutzt Edelmann Schwefelsäure, van Hasselt wasserfreie Phosphorsäure, die in einer dünnwandigen geschlossenen Glaskugel in das Gefäss gebracht wird und, nachdem man die Kugel durch Schütteln des Apparates zerbrochen hat, den Wasserdampf absorbirt. Mag auch die Benutzung der Schwefelsäure manche Unbequemlichkeiten mit sich führen, die angegebene Art und Weise, wie die Phosphorsäure hier verwandt wird, scheint mir den Gebrauch ganz besonders zu erschweren.

Wegen der engen Oeffnungen des Apparates macht endlich die Reinigung Schwierigkeiten; die Absorptions-

1) v. Hasse It, Beibl. 3. p. 697. 1879. 
mittel müssen mit Wasser ausgespült und das zurückbleibende Wasser mit Alkohol entfernt werden. Die Beseitigung der Alkoholdämpfe und die Füllung mit der auf ihren Feuchtigkeitsgehalt zu untersuchenden Luft ist dann noch durch Saugen auszuführen.

Um diese Uebelstände zu vermeiden, suchte ich einen Apparat zu construiren, bei welchem der luftdichte Verschluss nur durch Berührung ebengeschliffener, eingetalgter Flächen bewirkt wird, welcher gegen den Einfluss der äussern Luft ganz abgesperrt ist, keine Anwendung ron Absorptionsmitteln erfordert und nach dem Gebrauche leicht gereinigt werden kann.

Von zwei ungefähr $5 \mathrm{~cm}$ weiten und $12 \mathrm{~cm}$ hohen cylindrischen Glasgefässen mit flachabgebogenen, etwas verdickten Rändern von etwa $1 \mathrm{~cm}$ Breite wird die Randfläche eben abgeschliffen. Für jedes Gefäss ist ein mässig gewölbter, mit flachem und in ebensolcher Breite plangeschliffenem Rande versehener Glasdeckel bestimmt, in welchen etwas seitlich das eine $2 \mathrm{~cm}$ lange Ende eines rechtwinklig gebogenen Glasrohres von $2 \frac{1}{2}$ bis $3 \mathrm{~mm}$ Weite vertical eingeschmolzen ist. Die $3 \mathrm{~cm}$ langen horizontalen Theile beider Röhren sind durch gutes Patentgummirohr mit den rechtwinklig abgebogenen Enden eines ebenso weiten Manometerrohres, das zur Hälfte mit Rüböl gefüllt ist, möglichst nahe $\mathrm{zu}$ verbinden.

Werden die Gefässe, nachdem ihre ebenen Randflächen mit Talg oder mit einer fester bindenden Mischung von Talg, Wachs und Oel bestrichen und etwas Wasser in das eine gebracht worden, schnell geschlossen, so lässt sich der Druck des bis zur Sättigung neu entstehenden Wasserdampfes aus dem Manometerstand durch Rechnung ermitteln.

Durch das Andrücken der Deckel kann leicht eine in beiden Gefässen verschiedene Compression der Luft, also schon hierdurch eine Druckdifferenz beider Luftfüllungen verursacht werden. Ausserdem tritt in der Zeit zwischen dem Eingiessen des Wassers, dem Aufsetzen und Andrücken 
der Deckel eine Erhöhung des Feuchtigkeitsgehaltes der Luft im Apparate ein.

Zur Verhütung solcher Fehler ist nahe der Mitte jedes Deckels noch ein etwa $3 \mathrm{~cm}$ langes und $8 \mathrm{~mm}$ weites Rohrstück eingeschmolzen, dessen auf $4 \mathrm{~mm}$ verengtes Ende in einen flachen Rand ausläuft. Dieser Rand ist in einer Breite von wenigstens $4 \mathrm{~mm}$ abgeschliffen and mit einem getalgten Glasplättchen zu verschliessen. Um Luftblasen zu verhüten, wird nur die eine der beiden einander berührenden Flächen eingetalgt. Nach dem Auflegen müssen sie gleichmässig durchscheinend geworden sein.

Es kommt darauf an, dass die Verdunstung erst dann, wenn der Apparat geschlossen ist, dann aber auch möglichst rasch von statten geht. Ich stelle deshalb in das Gefäss eine aus zwei Lagen Filtrirpapier auf ein Korkscheibchen gewickelte und festgebundene, vielfach mit Oeffnungen versehene Rolle, welche halb so weit wie das Gefäss ist, und rom Boden bis nahe an den Deckel reicht.

Um Wasser in das Condensationsgefäss $\mathrm{zu}$ bringen, ohne dass der Feuchtigkeitsgehalt der Luft im Innern sich ändert, wird eine Kugelpipette benutzt, die 4 bis $5 \mathrm{~g}$ Wasser, etwas mehr als zur Sättigung der Papierrolle nöthig ist, in sich aufnehmen kann. Der $2 \mathrm{~cm}$ lange und $4 \mathrm{~mm}$ dicke Hals ist fest durch einen durchbohrten Korkpfropfen gesteckt; das etwa $3 \mathrm{~cm}$ lange Ausflussrohr verengt sich so, dass das Wasser nur heraustropfen kann. Nachdem die Pipette gefült, wird sie oben mit etwas Wachs geschlossen, sodass der Luftdruck auf die untere Oeffnung das Wasser zurückhält. So wird die Pipette mittelst des Korkes fest, aber undicht in das Deckelrohr unterhalb eingeklemmt.

Der Apparat wird nun in folgender Weise gebraucht. Nachdem die Gefässe am Rande gleichmässig dünn eingetalgt worden sind und, wie auch die übrigen Theile des Apparates, hinreichend lange im Beobachtungsraume gestanden haben, um seine Temperatur anzunehmen, wird eine Fliesspapierrolle, deren man mehrere zum Wechseln vorräthig 
hält, in das Condensationsgefäss gestellt, das Deckelpaar mit dem Manometer aufgelegt, wobei die Pipette sich in die Papierrolle senkt, und blos durch Gegenpressung der abstehenden Ränder festgesetzt. Befürchtet man, dabei den Apparat durch Berührung der Gefässwand in störender Weise erwärmt zu hahen, was möglichst zu vermeiden ist, so lässt man ihn jetzt noch mehrere Minuten stehen. Darauf wird das Deckelrohr des leeren Gefässes mit einem festanfgedrückten Glasplättchen geschlossen, die Wachsdecke der Pipette mit einer Nadel durchstochen und dann die Rohrmündung mit dem andern Glasplättchen sofort fest bedeckt.

Das Wasser tropft nun langsam auf die Korkscheibe der Papierrolle, welche letztere in 2 bis 3 Minuten völlig durchtränkt ist. Da das Wasser auf sehr grosser Fläche (von 100 bis $120 \mathrm{qcm}$ ) nach aussen und innen verdunstet, ist die Luft fast augenblicklich nach der Benetzung des Papiers mit Dampf gesättigt. Dies folgt daraus, dass bei der geringsten Abkühlung die Gefässwand sich innen mit Wasser beschlägt, und die bis dahin schnell steigende Manometerflüssigkeit fast plötzlich stehen bleibt. Lässt man dem Apparat dann noch einige Zeit, so zeigt sich unter Umständen eine geringe und deshalb meistens schwer zu constatirende Nachwirkung im Steigen, wenn das eingefüllte Wasser die herrschende Temperatur hatte. Diese Nachwirkung rührt daher, dass die zur Verdunstung nöthige Wärme dem feuchten Papier entzogen und dadurch eine geringe Druckverminderung verursacht wird, die sich erst dann ausgleicht, wenn die Temperatur im Innern wieder derjenigen der Umgebung gleich geworden ist. Die Nachwirkung muss übrigens bei Absorptionshygrometern in entgegengesetzter Weise und in viel höherem Grade bemorklich sein, weil meistens mehr Wasserdampf zu absorbiren ist, als zur Sättigung fehlt, und ausserdem die Contraction der Mischung von Schwefelsäure und Wasser eine noch weit beträchtlichere $W$ ärmeentwickelung veranlasst. Auch ist es rathsam, Wasser von der Lufttemperatur zu 
benutzen und den Apparat erst einige Zeit nach Befestigung der Pipette zu schliessen, um auf den Temperaturausgleich nicht zu lange warten zu müssen.

Dass die in den Poren des Fliesspapiers befindliche Luft durch Wirkung von Molecularkräften nur in durchaus unerheblichem Grade verdichtet sein kann, also auch nach ihrer Vertreibung durch das eindringende Wasser keinen Fehler im Manometerstande bewirkt, lässt sich daraus folgern, dass nach Einfüllung von Oel statt Wasser bei sorgfältig angestellten Versuchen sich keine Druckänderung am Manometer zeigte. Eine Absorption von Luft durch Wasser würde selbst dann kaum wahrnehmbar sein, wenn dasselbe ausgekocht wäre.

Die beim Schluss des Hygrometers vorzunehmenden Handgriffe haben keinen merklichen Einfluss auf den Manometerstand, wenn man dabei nur einigermassen geschickt verfährt. Selbstverständlich functionirt der Apparat an einem Orte, wo Luftströmungen von verschiedener Temperatur auf beide Gefässe wirken, nicht zuverlässig.

Zum Schutz gegen Temperaturungleichheiten erhält der Apparat in einem mit abnehmbarem Deckel versehenen leichten Holzkasten eine erhöhte Aufstellung. Etwa $8 \mathrm{~cm}$ über der Standfläche wird ein Brettchen mit runden Ausschnitten fest angebracht, deren Durchmesser demjenigen der Gefässe, und deren Abstand dem der beiden Deckel gleich ist. Dadurch wird das richtige Aufsetzen der letzteren erleichtert und zugleich den Gefässen ein fester Halt gegeben. Ein dritter Ausschnitt in dem Brettchen gestattet die Durchführung des Manometers und hinter demselben die feste Aufstellung eines Maassstabes, an welchem die Druckdifferenz bequemer abzulesen ist, als an einer auf dem engen Manometerrohr nur schwierig anzubringenden Theilung. Zur Ablesung des Manometers und eines daneben aufgestellten Thermometers wird aus der Vorderwand des Kastens ein Streifen von hinreichender Länge und Breite ausgeschnitten.

Die Enden des Manometerrohres und der mit ihnen 
zu verbindenden Knieröhren sind vor der Ausmündung etwas erweitert; die mit mässiger Spannung übergezogenen Gummirohrstücke halten dann fest uńd dicht genug und brauchen kaum noch seitwärts dieser Auftreibungen umbunden zu werden.

Der Druck ergibt sich aus dem Manometerstande durch folgende Rechnung. Das freie Volumen des Condensationsgefüsses (mit Subtraction des Volumens der Pipette und der Korkscheibe) bis zur Manometerflüssigkeit, wenn dieselbe noch keinen Höhenunterschied zeigt, sei $V$, das Volumen des andern Gefässes sei $V^{\prime}$ ccm. Im Moment der Absperrung sei der Barometerstand $b$, der Dunstdruck $e$ cm in Quecksilberhöhe, die Temperatur $t$. Die Manometerflüssigkeit habe das specifische Gewicht $s\left(=0,925-\frac{t}{1500}\right.$ für Rüböl), das des Quecksilbers sei $\sigma(=13,6)$. Zur Zeit der Ablesung betrage der Niveauunterschied im Manometer, dessen Querschnitt $q \mathrm{qcm}$ sei, $h \mathrm{~cm}$; die Temperatur sei $T$ und der entsprechende Druck des gesättigten Wasserdampfes gleich $E \mathrm{~cm}$.

Im ersten Gefässe ist dann der Druck:

im andern ist er:

$$
E+(b-e) \cdot \frac{V}{V+\frac{q h}{2}} \cdot \frac{1+\alpha T}{1+\alpha t}
$$

$$
b \cdot \frac{V^{\prime}}{V^{\prime}-\frac{q h}{2}} \cdot \frac{1+\alpha T}{1+\alpha t} \text {, wo } \alpha=0,003665 \text {. }
$$

Hieraus ergibt sich die Gleichung:

$$
\begin{aligned}
& \frac{h s}{\sigma}=E+(b-e) \cdot \frac{V}{V+\frac{q h}{2}} \cdot \frac{1+\alpha T}{1+\alpha t}-b \cdot \frac{V^{\prime}}{V^{\prime}-\frac{q h}{2}} \cdot \frac{1+\alpha T}{1+\alpha t}, \text { oder: }
\end{aligned}
$$

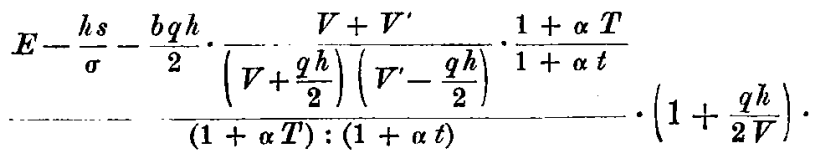

Bei den angegebenen Dimensionen darf der Factor $1+\frac{q h}{2 \vec{V}}$, sowie der Factor $\frac{1+\alpha T}{1+\alpha t}$ des letzten kleinen 
Gliedes im Dividendus weggelassen, $\left(V+\frac{q h}{2}\right) \cdot\left(V^{\prime}-\frac{q h}{2}\right)$ durch $V V$ und $(1+\alpha T):(1+\alpha t)$ im Divisor durch $1+\boldsymbol{\alpha} \cdot(\boldsymbol{T}-t)$ ersetzt werden. Es ist also:

$$
e=\frac{E-h e}{1+\alpha \cdot(T--t)} \text {. }
$$

Die Hülfsgrösse $c=\frac{s}{\sigma}+\frac{b q}{2} \cdot \frac{\nabla+V^{\prime}}{\bar{V} \bar{V}^{\prime}}$ kann für einen mittlern Barometerstand berechnet und als Constante des Apparates angesehen werden, da sogar bei den extremsten Barometerständen von $3 \mathrm{~cm}$ über oder unter dem mittlern Stande der aus dieser Annahme erwachsende Fehler von $h$ noch nicht ein Procent betragen kann. Ob der Divisor $1+\alpha \cdot(T-t) \mathrm{zu}$ beriücksichtigen oder zu vernachlässigen ist, lässt sich für den einzelnen Fall daraus beurtheilen, dass erst eine Temperaturänderung von , $T-t=2^{3} /{ }_{4}$ Grad einen Fehler von einem Procent für $e$ ergibt.

Wenn die Höhe $h$ in Millimetern gemessen wird, so ist sie durch Multiplication mit dem kleinen echten Bruche c jedesmal auf Quecksilberdruck zu reduciren. Diese Rechnung bleibt erspart, falls man einen Maassstab benutzt, dessen Intervalle $\frac{1}{10 . c} \mathrm{~mm}$ betragen. Die Einheiten dieses Maassstabes sind Zehntelmillimetern Quecksilberdruck äquivalent. Bei meinem Apparate ist $V=279,5, V^{\prime}=319,7$, $q=0,0560$; für den Mittelwerth $b=76$ ist also $\frac{1}{10 c}=1,215$. Ist der Maassstab für $s=0,925$ angefertigt, so hat man an der Ablesung $h$ noch die Correction $-\frac{t}{1500 \sigma c} \cdot h$, also $-\frac{t}{1680} \cdot h$ anzubringen, wenn man die Ausdehnung des Rüböls berücksichtigen will. Die nur sehr selten anzuwendende barometrische Correction für $h$ ist $+\frac{q}{2 c} \cdot \frac{V+V^{\prime}}{V V^{\prime}} \cdot(b-76) . h$, also $\frac{b-76}{440} \cdot h$.

Der Vorzug eines Manometers, welches mit einem geschlossenen Raume in Verbindung steht, vor einem nach 
aussen sich öffnenden liegt, abgesehen von der Unabhängigkeit von barometrischen Schwankungen, ganz besonders in dem zuverlässigen Verhalten gegen Temperaturänderungen, welche am offenen Manometer eine Druckänderung von mehr als $1 / 4 \mathrm{~mm}$ für $\frac{1}{10}$ Grad verursachen, dagegen bei dem in ein Gefäss mündenden Manometer gar nicht einwirken, wenn man dem Apparate Zeit zum Temperaturausgleich beider Gefässe lässt. Eine Temperaturänderung hat hierbei nur auf die Grösse von $E$ einen Einfluss, der in seinem Betrage viel geringer (bei $10^{\circ} 4^{1} / 2$, bei $20^{\circ}$ $2^{1} / 2$ mal) ist, also auch nur zu geringeren Fehlern Anlass geben kann. Auf ein sicheres Ergebniss ist also zu rechnen, wenn man zur Zeit des Schlusses der Gefässe eine erste, und, nachdem der Apparat geschlossen eine Zeit lang gestanden hat, noch eine zweite Temperaturablesung macht und dann die Manometerdifferenz abliest.

Am vortheilhaftesten ist es freilich, die Messungen in einem Raume anzustellen, in welchem die Temperatur keinem Wechsel ron Belang unterworfen ist. Wenn dies der Fall, so könnte man die Bedeckung des zweiten Gefässes unterlassen, den Apparat also mit offenem Manometer brauchen, ohne indess einen andern Vortheil zu erlangen als den, dass für die obige Formel (1) die Constante :

$$
c=\frac{s}{\sigma}+\frac{b q}{2 V}
$$

etwas kleiner, die Theilung des Maassstabes also etwas weiter ausfällt. Wenn hingegen eine Temperaturänderung eingetreten ist, so würde es sich nicht empfehlen, das Condensationsgefäss allein zu benutzen; denn die zur Berechnung von $e$ dienende Gleichung erhält dann, wie eine der obigen ähnliche Ableitung zeigt, sogar wenn der Barometerstand unverändert geblieben ist, die complicirtere Form:

$$
e=\frac{E+b \cdot a \cdot \frac{T-t}{1+\alpha t}-h \cdot\left(\frac{s}{\sigma}+\frac{b q}{2}\right)}{1+\alpha \cdot(T-t)} .
$$


Die Menge des verdunsteten Wassers, die dazu nöthige Wärme, die Temperatur- und Druckverminderung werden annähernd so berechnet. Die Dichtigkeit gesättigten Wasserdampfes für die Temperatur $T$ beträgt $D=\frac{0,0000106 E}{1+\alpha T}$, worin die Spannung $E$ in Centimetern zu nehmen ist. Das Gefäss enthält also im Zustande der Sättigung $D . V \mathrm{~g}$ Dampf; da der anfängliche Dunstdruck $e$ war, so mussten $\frac{0,0000106 V \cdot(E-e)}{1+\alpha} \mathrm{g}$ Wasser verdunsten. Dazu sind nach der Regnault'schen Formel für die latente Wärme des Dampfes $\frac{0,0000106 V \cdot(E-\epsilon)}{1+\alpha T} \cdot \frac{606,5-0,695 T}{1000}$ Calorien nöthig.

Für mittlere Verhältnisse, etwa für $T=17,5^{\circ}, E=1,49$, $e=0,89$, was 60 Proc. relativer Feuchtigkeit voraussetzt, und $V=280$ müssen hiernach $0,0017 \mathrm{~g}$ Wasser verdunsten, wozu 0,0010 Wärmeeinheiten erforderlich sind. Enthält nun das Fliesspapier etwa $4 \mathrm{~g}$ Wasser, und gäbe dieses allein die zur Verdunstung nöthige Wärme her, so würde es doch nur eine Temperaturerniedrigung von 0,25 Grad erfahren. Der auch blos zum Theil unter dieser wenig erniedrigten Temperatur entwickelte Dampf steht in zu geringem Quantitätsverhältnisse zu der Luft im Apparate, um dieselbe merklich abzukühlen. $\mathrm{Da}$ nun die directe Temperaturerniedrigung der Luft durch das nasse Papier auch nur allmählich vor sich gehen kann, und die inzwischen von der Gefässwand her zuströmende Wärme dieser $\mathrm{Ab}$ kühlung entgegenwirkt, so ist namentlich bei dünnwandigen Gefässen die Nachwirkung oft nicht erkennbar.

In ganz derselben Zusammenstellung dürfte der Apparat als Absorptionshygrometer zu benutzen sein, wenn man die Pipette mit Schwefelsäure gefüllt und mit Wachsvorschluss am Deckelrohr anbringt und auf geeignete Weise für möglichste Verbreitung der Säure Sorge trägt. Man füllt die Pipette mittelst eines Trichters, dessen enges Rohr bis in ihre Kugel hineinreicht, indem ihre untere Oeffnung zugehalten wird. Da hierbei die Luft durch die eindringende 
Säure sich nicht aus dem engen Ausflussrohr der Pipette vertreiben lässt, so ist die Füllung sehr bequem. - Die Dichtigkeit des Absorptionshygrometers ist wegen des äussern Ueberdruckes noch gesicherter.

Bei denselben Bezeichnungen wie oben ist der Druck im Absorptionsgefässe $(b-e) \cdot \frac{V}{V-\frac{q h}{2}} \cdot \frac{1+\alpha T}{1+\alpha t}$, im andern Gefässe $b \cdot \frac{V^{\prime}}{V^{\prime}+\frac{q h}{2}} \cdot \frac{1+\alpha T}{1+\alpha t}$, und es ergibt sich die Gleichung:

$$
\begin{aligned}
\frac{h s}{\sigma}= & b \cdot \frac{V^{\prime}}{V^{\prime}+\frac{q h}{2}} \cdot \frac{1+\alpha T}{1+\alpha t}-(b-e) \cdot \frac{V}{V-\frac{q h}{2}} \cdot \frac{1+\alpha T}{1+\alpha t}, \text { also: } \\
& \frac{h s}{\sigma}+\frac{b q h}{2} \cdot \frac{V+V^{\prime}}{\left(V-\frac{q h}{2}\right) \cdot\left(V^{\prime}+\frac{q h}{2}\right)} \cdot \frac{1+\alpha T}{1+\alpha t} \\
e=- & \frac{1+\alpha T):(1+\alpha t)}{(1+\alpha)} \cdot\left(1-\frac{q h}{2 V}\right),
\end{aligned}
$$

oder mit den erlaubten Vereinfachungen:

$$
e=\frac{h c}{1+\alpha \cdot(T-\bar{t})},
$$

worin dieselbe Hülfsgrösse $c=\frac{s}{\sigma}+\frac{b q}{2} \cdot \frac{V+V^{\prime}}{F V^{\prime}}$ auftritt, wie bei der Formel für das Condensationshygrometer. Die directe Ablesung des Druckes bis auf Zehntelmillimeter ist also durch denselben Maassstab ermöglicht.

Auch hier ergibt die Berechnung für den Gebrauch des blossen Absorptionsgefässes mit offenem Manometer, vorausgesetzt, dass der Barometerstand sich nicht geändert hat, die complicirtere Formel:

$$
e=\frac{b \alpha \cdot \frac{T-t}{1+\alpha t}+h \cdot\left(\frac{s}{\sigma}+\frac{b q}{2}\right)}{1+\alpha \cdot(T-t)},
$$

deren Anwendung auch nothwendig ist, um aus den Ablesungen. am Edelmann'schen Hygrometer die richtigen Werthe von $e$ zu berechnen.

Hinsichtlich der Leistungen habe ich den Apparat bei sehr verschiedenen Feuchtigkeitsverhältnissen, aber nur als 
Condensationshygrometer, mit einem in schwingender Bewegung erhaltenen guten Psychrometer verglichen und mit Beachtung der während dieser Versuche gemachten, hier angegebenen Erfahrungen eine sehr befriedigende Uebereinstimmung gefunden. Aus dem constanten Verhalten des Hygrometers muss ich schliessen, dass eine Diffusion von Wasserdampf durch das Gummirohr, welches van Hasselt deshalb am Edelmann'schen Hygrometer zu beseitigen für wünschenswerth hält, hier ganz und gar nicht vorkommt. Denn sogar während tagelangen Stehens meines Versuchsapparates zeigten sich in dem Stande des Manometers nur diejenigen Schwankungen, welche durch die von der Temperatur abhängige Aenderung der Dampfspannung bedingt waren. Wegen dieser Zuverlässigkeit dürfte das Hygrometer bei geschickter Behandlung sich besonders zur Ermittelung der je nach der Gebrauchsweise verschiedenen Psychrometerconstante eignen.

Hamburg, im October 1879.

\section{Ueber das durch electsische Entladumgen erzeugte Phosphorescenzlicht; von Eilhard Wiedemanu.}

Gelegentlich eines Referates in den Beiblättern 3. p. 526. 1879, über eine Arbeit von Crookes machte ich darauf aufmerksam, dass vielleicht die Platincyandoppelsalze unter dem Einflusse der von der negativen Electrode ausgehenden Entladungen eine orientirte Fluorescenz zeigen könnten.

Fast alle Platincyandoppelsalze zeigen unter dem Einflusse der Entladungen eine Fluorescenz, doch gelang es mir nicht an ihnen, so lange sie unzersetzt waren, eine Doppelfluorescenz nachzuweisen. Indess ergab sich eine Reihe anderer, der Beachtung werther Phänomene. $\left.{ }^{1}\right)$

1) Ueber dic Florescenzfarben selbst ist wäbrend meiner Versuchc eine Arbeit von Goldstein erschienen, auf die ich verweise (Wien. Ber. 80. 3. Juli 1879. 6 pp.) 